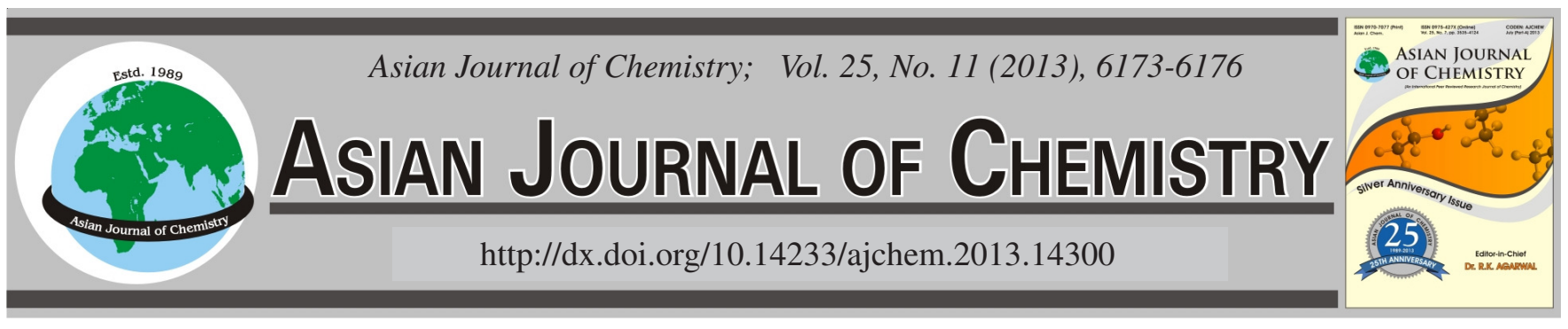

\title{
Structural and Magnetic Investigation of the Ferromagnetic ZnO:Co Nanotubes Fabricated by Electrophoretic Deposition Method
}

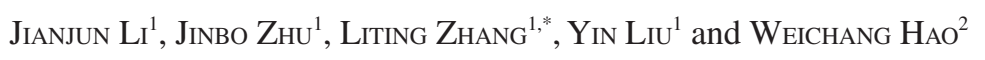

${ }^{1}$ Department of Materials Science and Engineering, Anhui University of Science and Technology, Huainan 232001, P.R. China

${ }^{2}$ Center of Materials Physics and Chemistry, Beihang University, Beijing 100083, P.R. China

*Corresponding author: Fax: +86 554 6668643; Tel: +86 554 6315463; E-mail: ljjhero4@ gmail.com

\begin{abstract}
$\mathrm{Zn}_{1-\mathrm{x}} \mathrm{Co}_{\mathrm{x}} \mathrm{O}$ nanotubes $(\mathrm{x}=0.01-0.07)$ have been controllable synthesized by electrophoretic deposition method using anodic aluminum oxide (AAO) as template. Careful structural characterization indicates that the prepared nanotubes are of poly-crystal wurtzite structure and composed with 8-15 nm nano-crystals. Magnetic investigation indicates the obtained nanotubes are of room-temperature ferromagnetic and the magnetism increases with the increase of Co doping. The magnetism of the nanotubes is much higher than that of the nanowires with similar dose of Co doping. Careful study indicates this difference of the magnetism is due to the surface-preferential Co distribution resulted by the electrophoretic deposition process.
\end{abstract}

Key Words: $\mathrm{Zn}_{1-\mathrm{x}} \mathrm{Co}_{\mathrm{x}} \mathrm{O}$ nanotubes, Electrophoretic deposition, Room-temperature ferromagnetism, Co distribution.

ᄂ - - - - - - - - - - - - - - - - - - - - - - - - -

\section{INTRODUCTION}

$\mathrm{ZnO}$ based diluted magnetic semiconductors (DMSs) have attracted great interests for their potential applications in semiconductor spintronics devices and thus, they have been receiving great attentions in recent years ${ }^{1}$. Current studies mainly focus on $\mathrm{Zn}_{1-\mathrm{x}} \mathrm{Co}_{\mathrm{x}} \mathrm{O}$ nanoparticles, thin films and bulk materials ${ }^{2-6}$. In contrast, the studies on one-dimensional (1 D) $\mathrm{Zn}_{1-\mathrm{x}} \mathrm{Co}_{\mathrm{x}} \mathrm{O}$ diluted magnetic semiconductors are still at a nascent stage despite very limited previous efforts, mostly focusing on $\mathrm{Zn}_{1-\mathrm{x}} \mathrm{Co}_{\mathrm{x}} \mathrm{O}$ nanowires ${ }^{7-9}$. However, the integration of diluted magnetic semiconductor materials into modern spintronics requires very low dimensions in order to make real use of the advantage offered by spins, where dimensionality and size are known to play a significant role in determining various properties of the systems. Therefore, synthesis and study of one dimensional (1 D) $\mathrm{Zn}_{1-\mathrm{x}} \mathrm{Co}_{\mathrm{x}} \mathrm{O}$ diluted magnetic semiconductors nanomaterials, especially nanotubes, are of great importance. Tubular form nanostructures are generally available in layered materials such as carbon nanotubes, thus, it seems more difficult to obtain the tubular nano-structure for the unlayered $\mathrm{ZnO}$ materials ${ }^{10}$. In this work, uniform morphology, highly ordered $\mathrm{Zn}_{1-\mathrm{x}} \mathrm{Co}_{\mathrm{x}} \mathrm{O}$ nanotube $(\mathrm{x}=0.01-0.07)$ arrays were controllable fabricated via electrophoretic deposition method with the aids of anodic aluminum oxide template. Their structure and magnetic property were carefully investigated and the mechanism of electrophoretic deposition is discussed.

\section{EXPERIMENTAL}

The anodic aluminum oxide template used in this work is purchased from Whatman co., England, with pore diameter of $200 \mathrm{~nm}$. All the reagents used in this experiment are of analytical grade, purchased from Shanghai Sinopharm Chemical Reagent Co. Ltd. The $\mathrm{Zn}_{1-\mathrm{x}} \mathrm{Co}_{\mathrm{x}} \mathrm{O}$ nanotubes were synthesized by an electrophoretic deposition method using using anodic aluminum oxide as template.

General procedure: Certain molar ratio of $\mathrm{Zn}(\mathrm{OAc})_{2} \cdot 2 \mathrm{H}_{2} \mathrm{O}$ and $\mathrm{Co}(\mathrm{OAc})_{2} \cdot 4 \mathrm{H}_{2} \mathrm{O}$ were dissolved in $200 \mathrm{~mL}$ of dimethyl sulfoxide. Then $50 \mathrm{~mL}\left(\mathrm{CH}_{3}\right)_{4} \mathrm{NOH} \cdot 4 \mathrm{H}_{2} \mathrm{O}$ solution was added to the previous solution to form stable colloid. Electrophoretic deposition was performed in a bi-electrodes system under constant voltage mode. An anodic aluminum oxide template membrane, with a surface sputter-coated with gold, acted as working electrode (negative electrode) and a reeled Pt thread acted as counter electrode. For $\mathrm{Zn}_{1-\mathrm{x}} \mathrm{Co}_{\mathrm{x}} \mathrm{O}$ nanotube electrophoretic deposition, a direct potential of 4.5-5.0 V was applied on the electrodes and sustained for $45 \mathrm{~min}$. Then the prepared samples were annealed at $550{ }^{\circ} \mathrm{C}$ for $6 \mathrm{~h}$ in air atmosphere. Careful wet chemical etching with $1 \mathrm{~mol} / \mathrm{L} \mathrm{NaOH}$ was required to remove the alumina membrane before structural and magnetic characterization. The experimental detail can be found elsewhere ${ }^{7}$.

Detection method: The scanning electron microscopy image and back-scattered electron image (BEI) were obtained 
by a JSM-5600LV SEM equipped with energy dispersive spectrometer. The transmission electronic microscopy and high resolution transmission electronic microscopy images were obtained by a JEM-2100F TEM operated at $200 \mathrm{KV}$. The structure of the nanowires were characterized by a Rigaku D/Max-2200 $\mathrm{X}$-ray diffracton with $\mathrm{CuK}_{\alpha}(1.54 \AA)$ line. The Raman spectra were obtained with JY-T64000 micro-Raman spectrometer in the backscattering geometry excited by $\mathrm{Ar}^{+}$laser $532 \mathrm{~nm}$ line. The magnetic properties were characterized by a vibrating sample magnetometer (VSM, LakerShore 7410) at $300 \mathrm{~K}$.

\section{RESULTS AND DISCUSSION}

Fig. 1 shows the SEM images of the $\mathrm{Zn}_{1-\mathrm{x}} \mathrm{Co}_{\mathrm{x}} \mathrm{O}(\mathrm{x}=0.05)$ nanotubes. As shown in the SEM images (Fig. 1a), the prepared nanotubes have homogeneous morphology and orderly distribution. The length of the nanotubes is tens of microns, corresponding to the thickness of the anodic aluminum oxide template. The EDS spectrum (not shown) suggests that the nanotubes are only composed of $\mathrm{Zn}, \mathrm{Co}$ and $\mathrm{O}$ elements. The atomic ratio of $\mathrm{Zn}$ to $\mathrm{Co}$ is measured as 81:4, close to the nominal Co doping ratio. Careful EDS surface scan results (Fig. 1c-e) indicate the homogenous distribution of $\mathrm{Zn}$ and Co element in the nanotubes. From the BEI image (Fig. 1b), the inner structure of the nanotubes is clearly observed. The filled section of nanotube (near the bottom) is brighter than the rest (hollow) part. The brightest points at the bottom area are the sputtering-coated gold layer, acting as working electrode for the electrophoretic deposition. The presence of the bottom filled nanotubes implies the electrophoretic deposition may be a bottom-up process.

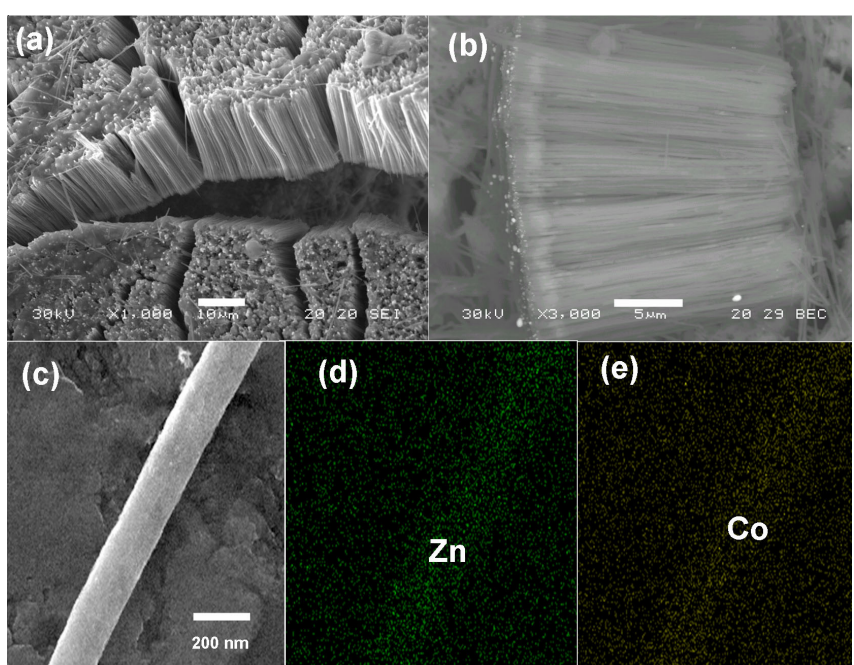

Fig. 1. (a), (b) SEM and BEI images of $\mathrm{Zn}_{0.95} \mathrm{Co}_{0.05} \mathrm{O}$ nanotubes. (c), (d), (e) show the SEM image, Zn element and Co element EDS surface scan image of a single $\mathrm{Zn}_{0.95} \mathrm{Co}_{0.05} \mathrm{O}$ nanotube, respectively

Fig. 2a shows the TEM image of the $\mathrm{Zn}_{0.95} \mathrm{Co}_{0.05} \mathrm{O}$ nanotubes. It is seen that the diameter of $\mathrm{Zn}_{0.95} \mathrm{Co}_{0.05} \mathrm{O}$ nanotubes is $190 \mathrm{~nm}$ around, a little thinner than that of the anodic aluminum oxide template pores (200 nm around), which is owing to the densification of the gel during annealing process $^{10,11}$. Half-filled nanotubes are clearly seen in the view scope. Considering the BEI results, the bottom-up process of the electrophoretic deposition can be concluded. HRTEM

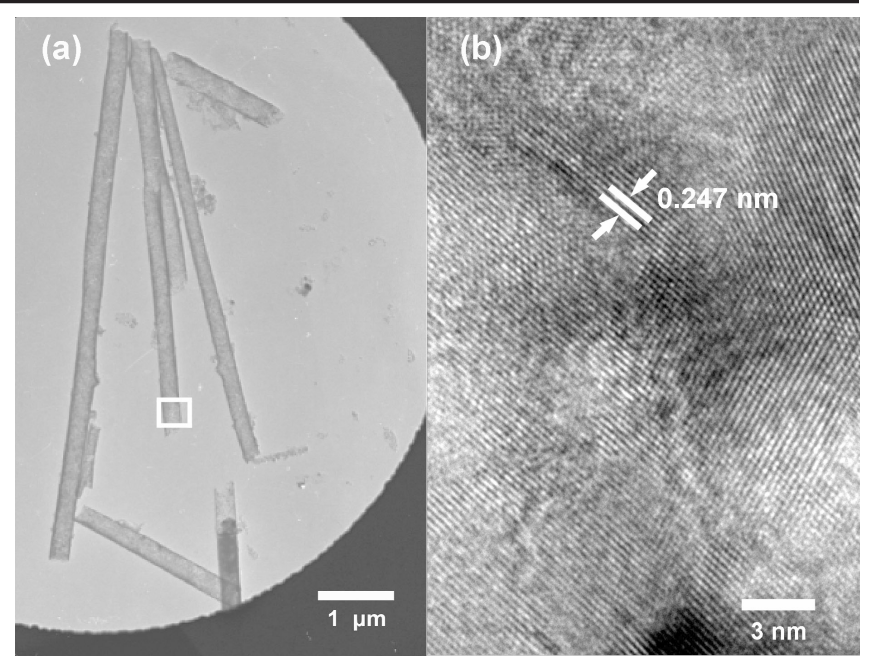

Fig. 2. (a) TEM, (b) HRTEM images of $\mathrm{Zn}_{0.95} \mathrm{Co}_{0.05} \mathrm{O}$ nanotubes

investigation (Fig. 2b) indicates the nanotubes are composed of lots of 8-15 nm width nano-crystallines. The labeled diffraction fringe distances is $0.247 \mathrm{~nm}$, well corresponding to the interplanar distance of (101) planes of Wurtzite $\mathrm{ZnO}$, which indicates the nano-crystallines in the nanotubes are of Wurtzite structure. No second-phase structure can be found in the view scope. Some amorphous structure is observed between $\mathrm{Zn}_{0.95} \mathrm{Co}_{0.05} \mathrm{O}$ nano-crystallines.

Fig. 3 shows the XRD spectra of the $\mathrm{Zn}_{1-x} \mathrm{Co}_{x} \mathrm{O}$ nanotubes. It is seen all the nanotubes are similar to the hexagonal structure wurtzite ZnO (JCPDS number: \#65-3411). No second-phase composition could be found in all the XRD spectra. This suggests the obtained nanotubes keep the Wurtzite structure of hexagonal $\mathrm{ZnO}$ and the doped $\mathrm{Co}^{2+}$ ions mainly replace the $\mathrm{Zn}^{2+}$ ions in $\mathrm{ZnO}$ lattice, which is also supported by the Raman spectra and UV-visible absorption (not shown). As shown in Fig. 4, all the Raman peaks of the samples are in good coincident with the typical Raman spectra of the Wurtzite $\mathrm{ZnO}^{12,13}$. The peaks at 97, $201333,437,536,578,666,985,1146$ and around $536 \mathrm{~cm}^{-1}$ refer to $\mathrm{E}_{2 \mathrm{~L}}, 2 \mathrm{E}_{2 \mathrm{~L}}, \mathrm{E}_{2 \mathrm{H}}-\mathrm{E}_{2 \mathrm{~L}}, \mathrm{E}_{2 \mathrm{H}}, 2 \mathrm{~B}_{1 \mathrm{~L}}, \mathrm{~A}_{1 \mathrm{~L}}$ or $\mathrm{E}_{1 \mathrm{~L}}$ or $\mathrm{LO}, \mathrm{TA}+\mathrm{LO}, 2 \mathrm{TO}, 2 \mathrm{~A}_{1 \mathrm{~L}}$ or $2 \mathrm{E}_{1 \mathrm{~L}}$ and a multiphonon mode, respectively. The intensities of all the peaks decrease with the increasing of Co doping. It suggests that the doped $\mathrm{Co}^{2+}$ ions mainly occupy the $\mathrm{Zn}^{2+}$ sites and reduce the translational symmetry of the allowed phonons of the host $\mathrm{ZnO}$ lattice. The broad Raman peak at $536 \mathrm{~cm}^{-1}$ around is originated from the sp-d exchange in $\mathrm{ZnO}$ :Co system ${ }^{14}$, which is thought to be one of the necessary conditions for achieving ferromagnetism in diluted magnetic semiconductors materials ${ }^{15}$. The increase of its intensity with the increase of Co dopant implies the magnetism of the nanotubes may increase with Co doping, which is proved by the magnetic investigation.

Fig. 5 shows the magnetization of the $\mathrm{Zn}_{1-x} \mathrm{Co}_{\mathrm{x}} \mathrm{O}$ nanotubes measured by VSM at $300 \mathrm{~K}$. All the $\mathrm{Zn}_{1-\mathrm{x}} \mathrm{Co}_{\mathrm{x}} \mathrm{O}$ nanotubes show room-temperature ferromagnetism. Their magnetism increases with the increasing of the Co doping for the increase of Co concentration, which improves the ferromagnetic coupling between Co ions ${ }^{12}$. It is noted that the ferromagnetism of the nanotubes is much higher than that of the nanowires with same dose Co dopant. As shown in the inset of Fig. 5, the ferromagnetisms of $\mathrm{Zn}_{0.95} \mathrm{Co}_{0.05} \mathrm{O}$ nanotubes and nanowires are 


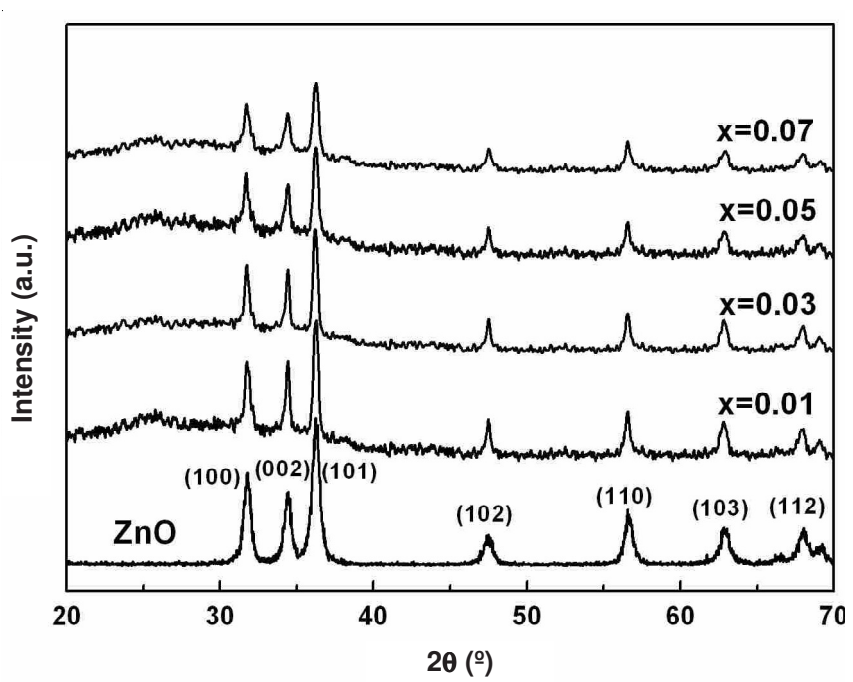

Fig. 3. XRD spectra of $\mathrm{Zn}_{1-\mathrm{x}} \mathrm{Co}_{\mathrm{x}} \mathrm{O}$ nanotubes and pure $\mathrm{ZnO}$ powders

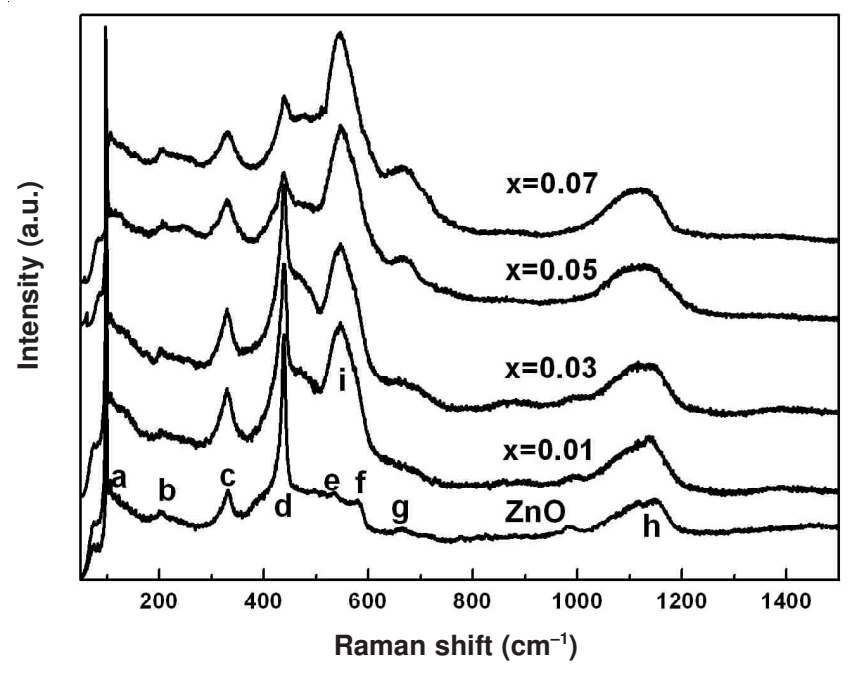

Fig. 4. Raman spectra of $\mathrm{Zn}_{1-\mathrm{x}} \mathrm{Co}_{\mathrm{x}} \mathrm{O}$ nanotubes and pure $\mathrm{ZnO}$ powders

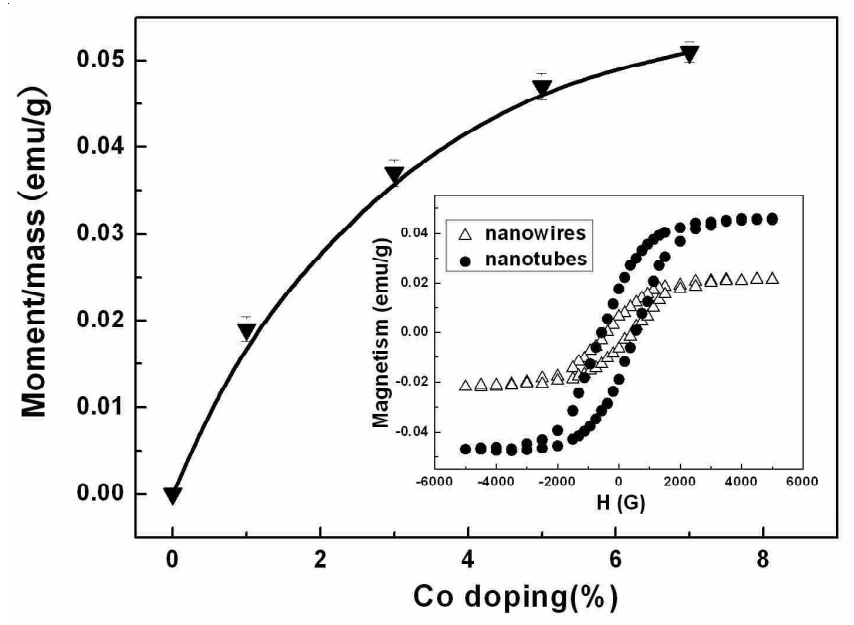

Fig. 5. Evolution of magnetism of $\mathrm{Zn}_{1-\mathrm{x}} \mathrm{Co}_{\mathrm{x}} \mathrm{O}$ nanotubes as a function of Co doping detected by VSM at $300 \mathrm{~K}$. The inset shows the M-H curves of $\mathrm{Zn}_{0.95} \mathrm{Co}_{0.05} \mathrm{O}$ nanotubes and nanowires

0.047 and $0.022 \mathrm{emu} / \mathrm{g}$, respectively. The magnetism difference between nanotubes and nanowires may be derived from the surface-preferential of the doped Co ions. Since the formation of $\mathrm{ZnO}$ : Co colloid is a self-purification process ${ }^{16,17}$, the Co concentration at the surface area is higher than that in the inner $\operatorname{part}^{2,18}$. Nanotubes have a larger specific surface area than that of nanowires, thus they have a larger Co-rich area. According to the bound magnetic polarons (BMPs) theory proposed by Coey et al. ${ }^{19}$ shallow donor electrons bound to defects formed BMPs and created a spin-split impurity band. The BMPs played a critical role in mediating the ferromagnetic interaction between the transition metal ions. The surface-preferential distribution of Co enriches the magnetic element at the surface area. The higher Co concentration in $\mathrm{Zn}_{0.95} \mathrm{Co}_{0.05} \mathrm{O}$ nanotubes surface is beneficial to BMP formation and the ferromagnetic coupling between Co ions ${ }^{19,20}$, leading to the higher ferromagnetism of the nanotubes.

According to the discussion above, the ferromagnetism of $\mathrm{Zn}_{1-x} \mathrm{Co}_{\mathrm{x}} \mathrm{O}$ is closely related to its morphologies, which depends on the electrophoretic deposition condition. In this work, the $\mathrm{Zn}^{2+}: \mathrm{Co}^{2+}$ colloidal particles were carefully tuned to be positively charged (inside of the slip plane). During the electrophoretic deposition process, the colloidal particles with a little adsorbed liquid moved to the cathode under external electric field. Firstly, a colloidal tube, with a thickness of several colloidal particle layers, formed on the pore surface due to the adsorption effect of the anodic aluminum oxide template inner wall. Then the colloidal tubes gelated and were filled from the bottom to the top by the latter colloidal particles. The properties of the tubes and the filled length were determined by the electrophoretic deposition potential, deposition time and the $d$ potential of the colloidal particles. If the deposition potential, time and the $\mathrm{pH}$ value were properly tuned, both $\mathrm{Zn}_{1-\mathrm{x}} \mathrm{Co}_{\mathrm{x}} \mathrm{O}$ nanotubes and nanowires could be fabricated.

\section{Conclusion}

$\mathrm{Zn}_{1-\mathrm{x}} \mathrm{Co}_{\mathrm{x}} \mathrm{O}(\mathrm{x}=0.01-0.07)$ nanotubes have been controllable synthesized by electrophoretic deposition method using anodic aluminum oxide as template. Magnetic investigation indicates all the $\mathrm{Zn}_{1-\mathrm{x}} \mathrm{Co}_{\mathrm{x}} \mathrm{O}$ nanotubes samples are of room-temperature ferromagnetism. The magnetism of the nanotubes is much higher than that of the corresponding nanowires for higher Co concentration at the surface area. The mechanism of the electrophoretic deposition is discussed.

\section{ACKNOWLEDGEMENTS}

This work is supported by the Open Project of Key Laboratory for Magnetism and Magnetic Materials of the Ministry of Education, Lanzhou University (Grant No. LZUMMM2012001) and the National Natural Science Foundation of China (Grant No. 51208003).

\section{REFERENCES}

1. T. Jungwirth, J. Sinova, J. Mašek, J. Kucera and A.H. MacDonald, Rev. Mod. Phys., 78, 809 (2006).

2. J. Li, W. Hao, H. Xu and T. Wang, J. Appl. Phys., 105, 053905 (2009).

3. B.B. Straumal, A.A. Mazilkin, S.G. Protasova, A.A. Myatiev, P.B. Straumal, E. Goering and B. Baretzky, Thin Solid Films, 520, 1192 (2011).

4. C. Wang, B. Man, M. Liu, C. Chen, S. Jiang, S. Yang, S. Xu, X. Gao and B. Hu, Adv. Condens. Mater. Phys., 2012, 363981 (2012).

5. L.J. Zhang, J.Q. Wang, J. Li, J. Zhou, W.P. Cai, J. Cheng, W. Xu, G. Yin, X. Wu, Z. Jiang, S. Zhang and Z.-Y. Wu, Chem. Commun., 48, 91 (2012).

6. D. Seghier and H.P. Gislason, J. Mater. Sci.: Mater. Electron., 22, 1400 (2011).

7. J. Li, L. Zhang, J. Zhu, Y. Liu and W. Hao, Mater. Lett., 87, 101 (2012). 
8. B.D. Yuhas, D.O. Zitoun, P.J. Pauzauskie, R. He and P. Yang, Angew. Chem. Int. Ed., 118, 434 (2006).

9. S. Han, D. Zhang and C. Zhou, Appl. Phys. Lett., 88, 133109 (2006).

10. Z.L. Xiao, C.Y. Han, U. Welp, H.H. Wang, W.K. Kwok, G.A. Willing, J.M. Hiller, R.E. Cook, D.J. Miller and G.W. Crabtree, Nano Lett., 2, 1293 (2002).

11. L. Liu, W. Lee, Z. Huang, R. Scholz and U. Gösele, Nanotechnology, 19, $335604(2008)$

12. J.M. Calleja and M. Cardona, Phys. Rev. B, 16, 3753 (1977).

13. R. Cuscó, E. Alarcón-Lladó, J. Ibáñez, L. Artús, J. Jiménez, B. Wang and M.J. Callahan, Phys. Rev. B, 75, 165202 (2007).
14. H. Zhou, L. Chen, V. Malik, C. Knies, D.M. Hofmann, K. P. Bhatti, S. Chaudhary, P.J. Klar, W. Heimbrodt, C. Klingshirn and H. Kalt, Phys. State Solidii (a), 204, 112 (2007).

15. K. Ando, Science, 312, 1883 (2006).

16. D.J. Norris, A.L. Efros and S.C. Erwin, Science, 319, 1776 (2008).

17. G.M. Dalpian and J.R. Chelikowsky, Phys. Rev. Lett., 96, 206802 (2006).

18. P. Lommens, K. Lambert, F. Loncke, D. De Muynck, T. Balkan, F. Vanhaecke, H. Vrielinck, F. Callens and Z. Hens, Chem. Phys. Chem., 9, 484 (2008).

19. J. Coey, M. Venkatesan and C. Fitzgerald, Nature Mater., 4, 173 (2005).

20. D. Iusan, B. Sanyal and O. Eriksson, J. Appl. Phys., 101, 109H101 (2007). 